\title{
Marking technique in strabismus correction
}

\section{Técnica de marcaje en cirugía de corrección de estrabismo}

\author{
Mariana E. López-Hernández, and José F. Pérez-Pérez*
}

Instituto Mexicano de Oftalmología I.A.P., Querétaro, Querétaro, Mexico

\begin{abstract}
Background: Despite international efforts to conduct a standardized surgical safety protocol, there are still errors such as operating on the wrong eye. There is a universal protocol that was implemented back in July 2004 by the joint commission that seeks to prevent errors. It includes three basic points: preoperative verification, marking of the surgical site, and immediate waiting time before the incision. Objective: To propose a marking method that allows us, in addition to knowing which eye to operate on, to have both a horizontal and vertical surgical approach on the patient's skin to have more certainty on the procedure to be performed. Method: Description of the marking technique in patients with strabismus based on the type of deviation, and surgery proposed in each muscle and patient. Results: Several examples of the surgical plan are presented with photos and diagrams.
\end{abstract}

Keywords: Strabismus. Marking. Surgery. Prevention.

\section{Resumen}

Antecedentes: A pesar de los esfuerzos internacionales por realizar un protocolo estandarizado de seguridad quirúrgica, aún existen errores, como operar el ojo incorrecto. Existe un protocolo universal, implementado en julio de 2004 por una comisión conjunta, que busca prevenir errores e incluye tres puntos básicos: verificación preoperatoria, marcaje del sitio quirúrgico y tiempo de espera inmediato antes de la incisión. Objetivo: Proponer un método de marcaje que permita, además de saber qué ojo operar, tener el planteamiento quirúrgico tanto horizontal como vertical sobre la piel del paciente para aportar mayor certeza del procedimiento a realizar. Método: Descripción de la técnica de marcaje en el paciente con estrabismo, de acuerdo con el tipo de desviación, y de la cirugía propuesta para cada músculo y paciente. Resultados: Se presentan varios ejemplos del plan quirúrgico, con fotos y esquemas.

Palabras clave: Estrabismo. Marcaje. Cirugía. Prevención.

\section{Introduction}

Marking the surgical site is essential and it is part of the procedures of any surgical event. Also, verifying the eye to be operated on by the nursing staff and the treating physician is also part of the patient's safety procedures, to prevent procedural mistakes as much as possible such as operating on the wrong eye or performing the wrong procedure.

\section{Correspondence:}

*José F. Pérez

Av. Armando Birlain Shaffler S/N,

Date of reception: 18-06-2021

Centro Sur, 76090 Santiago de Querétaro, Qro., Mexico

E-mail: ferchus67@live.com.mx

(http://creativecommons.org/licenses/by-nc-nd/4.0/).
Date of acceptance: 08-10-2021

DOI: 10.24875/RMOE.M21000204
Available online: 31-01-2022

Rev Mex Oftalmol (Eng). 2022;96(1):14-21

www.rmo.com.mx 
Despite international efforts to conduct a standardized surgical safety protocol, there are still errors such as operating on the wrong eye. There is a universal protocol that was implemented back in July 2004 by the joint commission that seeks to prevent errors. It includes three basic points: preoperative verification, marking of the surgical site, and immediate waiting time before the incision ${ }^{1,2}$.

The specialty of ophthalmology shows a high rate of surgical errors, among them, the wrong surgical site ranks in first (the rate is 1.8 for every 10000 cases) ${ }^{2}$. Based on the occurrence report program and patient follow-up from the New York State Health Department, the rate of error was 6.9 for every 100000 patients and 4 for every 10000 patients in strabismus surgeries ${ }^{2}$.

When conducting a bibliographical review, the following risk factors were found for the occurrence of these errors: lack of communication between surgeon and the staff involved, lack of verification prior to the procedure to be performed, distractions, and incomplete assessments of the patient, among others ${ }^{3,4}$.

Back in August 2014, the American Academy of Ophthalmology working group published a series of recommendations that suggested performing actions prior to the day of the surgery such as getting the informed consent signed, and on the day of the surgery, verifying the eye and procedure to be performed, paying special attention to procedures based on preoperative estimates like placing an intraocular lens ${ }^{5}$.

There is another universal protocol, published in 2003 by the Joint Commission, that highlights the importance of 3 key aspects: preoperative verification, marking the site, and calling a «time out» in the operating room.

In a study conducted in the year 2011 in which strabismus surgeons were surveyed during a meeting held by the American Association for Pediatric Ophthalmology and Strabismus (AAPOS) to know the causes of errors during surgery, the following were recognized as the leading causes of errors: mistaking the type of deviation (esotropia or exotropia), error in the surgical procedure (weakening or strengthening) $(29.8 \%)$, eyeball torsion $(17.5 \%)$, which mainly leads to an involuntary operation in the inferior rectus rather than in the target internal rectus muscle, and lack of attention or distraction $(16.7 \%)$. Using more than one operating room $(P=.02)$ plus not marking the eye muscles prior to the operation $(P=.03)$ were associated with higher chances of error. In such study, marking was only performed to indicate whether it was weakening or reinforcement, and at no time was indicated whether there was muscle displacement or whether any other associated surgical techniques were performed ${ }^{3}$.

Gibbs admits that it has not passed enough time yet since the introduction of the universal protocol to determine its effectiveness reducing the rate of surgeries performed in the wrong site, speaking of neurosurgery specifically; however, he is aware and admits that implementing the rules and protocols has an impact on the way surgeries start, and improves communication among members of the surgical team ${ }^{4,6,7}$.

An additional recommendation is to prevent distractions, interruptions, and constant changes or different procedures on the day of the surgery ${ }^{3,8,9}$.

The treating surgeon should have a lead role emphasizing the importance of preparing the facilities and having everything ready before the surgery is performed, thus preventing any possible complications from happening and creating quick communications that should make it possible, in case of emergency or need for a change, to implement the appropriate measures the best possible way ${ }^{3,6,9}$.

A follow-up protocol should be established so that the nursing staff and the treating physician and physicians-in-training are taught to minimize previous errors before, during, and after surgery. In a study conducted at an orthopedic teaching hospital where the errors made in the surgeries performed were analyzed, before and after training the staff, based on the statistics, and working on each error, standards, and protocols were implemented to reduce the rate of errors. The rate went down from $18.7 \%$ prior to the study to $5.9 \%$ after training $(P=.0001)^{10}$.

The objective of this study is to propose a marking method that should make it possible, in addition to knowing which eye to operate on, to have both the horizontal and vertical surgical approaches planned, as well as the planning of special cases, on the patient's skin to have one additional element of certainty on the procedure to be performed ${ }^{11}$.

\section{Method}

A descriptive, prospective study conducted in the pediatric ophthalmology and strabismus unit of the Mexican Institute of Ophthalmology I.A.P.

The study included patients with a diagnosis of strabismus scheduled for surgical correction from January through March 2019. The size of the sample was at convenience, and a total of 23 patients were recruited who had been assigned following the department's 
routine scheduling system. Patients with a skin condition who did not allow marking on the skin and those who did not wish to participate in the protocol were excluded. All patients signed the routine informed consent (the parents or the corresponding tutors of the pediatric patients). At the end of the study, the surgeon was surveyed to assess his opinion on the marking technique.

Initially, the eye to be operated on was marked by the nursing staff in the OR area as part of the safety standards established, by placing a mark in the shape of a wheel or dot on the forehead above the eye or both eyes in the very frequent case of strabismus in which both eyes are operated on. Afterward, the surgeon marked on the patient's skin the site of the muscle to be operated on in its functional site based on the scheme that is used to register strabological disorders (Fig. 1). Marking was performed once the patient had been changed inside the OR prior to the anesthesia procedure by the surgeon with the help of his assistant. A fine-tipped, permanent black marker was used (Fig. 2) so that the marking would not be erased with the surgical cleaning. Marking was erased with saline wipes at the end of the procedure when the skin on the surgical site was cleaned.

This is the marking technique proposed:

- In horizontal surgery:

- Correction of horizontal strabismus whether esotropia and exotropia: action site of internal and external rectus muscles.

- Marking on the horizontal muscle region to be operated on and differentiation between a strengthening procedure and a weakening one:

- Strengthening: positive sign marking with (+).

- Weakening: negative sign marking (-).

- In vertical surgery.

- Correction of hypertropia or hypotropia: action site of the inferior and superior rectus muscles.

- Marking on the vertical muscle region to be operated on:

- Strengthening: positive sign marking (+).

- Weakening: negative sign marking (-).

- To know whether muscular displacement should be performed:

- Arrow pointing up or down on the muscular region to be operated on, indicating displacement direction.

- To distinguish the oblique muscles to be operated on, negative sign (-):

- Marking the functional region of the inferior oblique muscle.

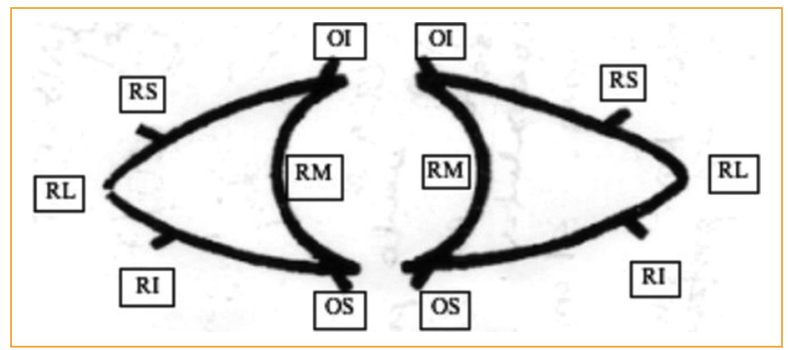

Figure 1. Diagram showing the functional regions of extraocular muscles corresponding to the marking sites on the patient's skin. OI, inferior oblique muscle; OS, superior oblique muscle; Rl, inferior rectus; RL, lateral rectus; RM, medial rectus; $\mathrm{RS}$, superior rectus.

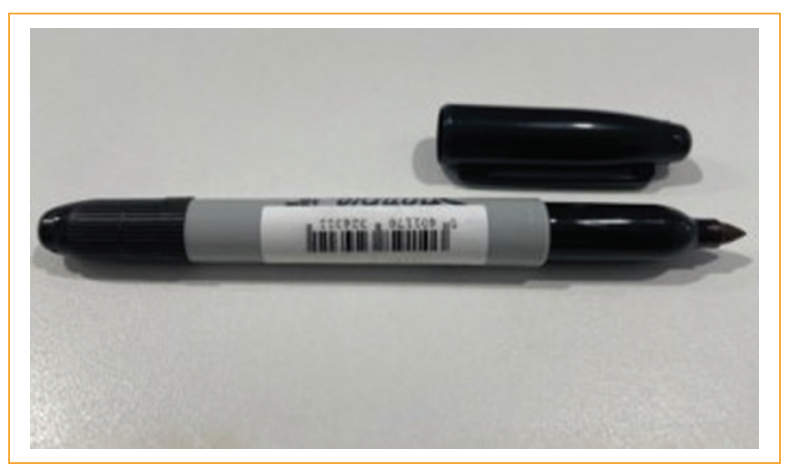

Figure 2. Permanent marker used to mark on the patient's skin.

- Marking the functional region of the superior oblique muscle.

- Surgery in special cases:

- Technique for placing Faden suture: negative sign to mark weakening with central, cross-sectional line indicative of Faden suture.

- Muscular transpositions: a curved arrow is pointing up or down in the direction of transposition (Fig. 3).

Finally, at the end of the period, the expert surgeon responsible for the marking and the surgeries performed is surveyed to know his opinion on the marking technique use (its ease, comfort, importance, and usefulness), as well as his recommendations.

\section{Results}

The study went on for three months and included 23 patients who had been operated on at the Instituto Mexicano de Oftalmología I.A.P. unit of pediatric ophthalmology and strabismus. Patients were marked on the surgical sites and the muscles to be operated on 


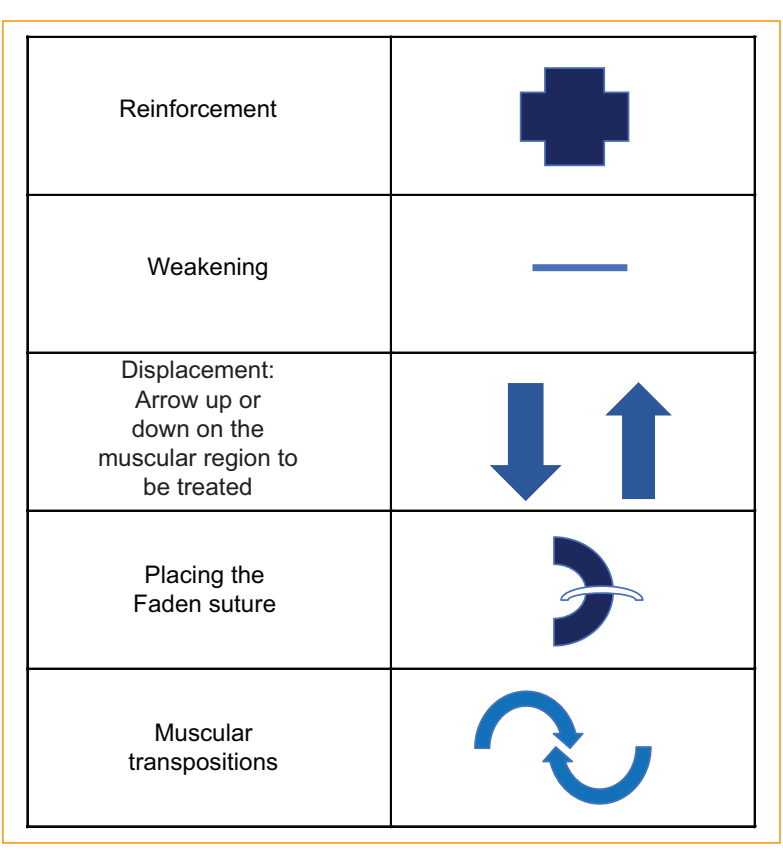

Figure 3. Drawings used for marking.

based on their action sites, in the manner explained above.

Out of the 23 patients included, $56.52 \%$ were women, and $43.48 \%$ were men (Fig. 4). The age range went from 2 to 66 years, with a mean age of 17.5 years. Strabological diagnoses were different: Ten patients had exotropia, nine esotropia, and four were diagnosed with other conditions (Table 1).

Of all surgeries performed, $56.52 \%$ were performed on both eyes (Fig. 5). Weakening was the most common procedure of all $(51 \%)$ followed by strengthening $(28.57 \%)$, supradisplacement $(11.90 \%)$, and infradisplacement $(4.76 \%)$, Faden suture $(9.52 \%)$, and eventually inferior oblique muscle transposition (4.76\%) (Fig. 6).

\section{Examples of the marking technique}

Five cases are presented with strabological examination, eye movement abnormality, and intraoperative marking of surgical plan.

Case 1: diagnosis of residual right monocular exotropia (Fig. 7)

- Examination:

-50 DP left monocular exotropia.

- Ductions and versions: hyperfunction of inferior oblique muscles 2+, V syndrome, limitation -1 left eye abduction.

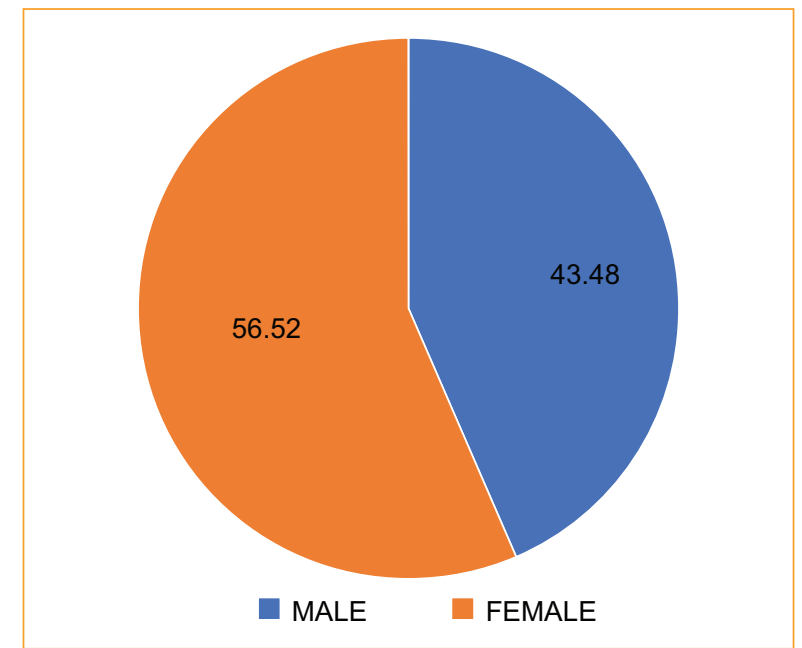

Figure 4. Distribution by sex.

Table 1. Diagnosis table

$\begin{array}{ll}\text { Exotropies } & - \text { Post-surgical exotropia } \\ & - \text { Secondary exotropia + A syndrome } \\ & - \text { Secondary exotropia. (2 cases) } \\ & - \text { Exophoria/tropia + V syndrome (2 cases) } \\ & - \text { Decompensated exophoria/tropia (3 cases) } \\ & - \text { Decompensated exophoria/tropia + A syndrome } \\ \text { Esotropias } & - \text { Congenital esotropia. (5 cases) } \\ & - \text { Congenital esotropia }+ \text { A syndrome (3 cases) } \\ & - \text { Variable angle esotropia } \\ \text { Others } & - \text { Sixth nerve palsy of the right eye } \\ & - \text { Fourth cranial nerve palsy of the right eye } \\ & - \text { Left eye Duane syndrome } \\ & - \text { Nystagmus with compensating position of } \\ & \text { head }+ \text { exotropia }\end{array}$

- Surgical plan: retroinsertion of both external recti with supradisplacement, and strengthening of both internal recti.

Note: in this patient, the markings on the eye to be operated on look like an " $X$ ", yet today routine clinical practice is to follow the standard of using a circle or a dot.

Case 2: diagnosis of right eye monocular esotropia of variable angle (Fig. 8)

\section{- Examination:}

- 15-30 DP right monocular esotropia.

- Ductions and versions: hyperfunction of the inferior oblique muscles +1 , $V$ syndrome, limitation -1 right eye abduction. DVD +1 right eye.

- Surgical plan: weakening of right eye internal rectus + Faden suture.

Note: in this patient, the markings on the eye to be operated on look like an " $X$ ", yet today routine clinical 


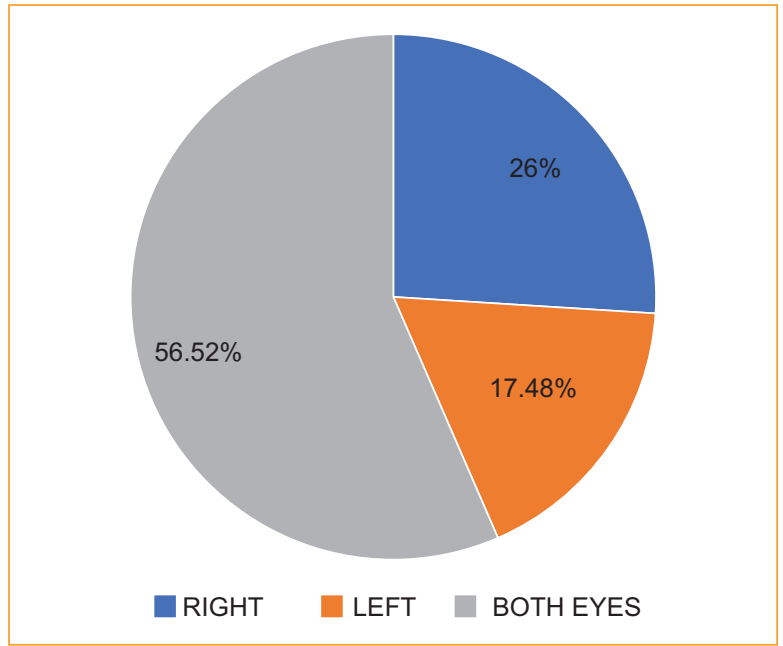

Figure 5. Distribution by eye to be operated on.

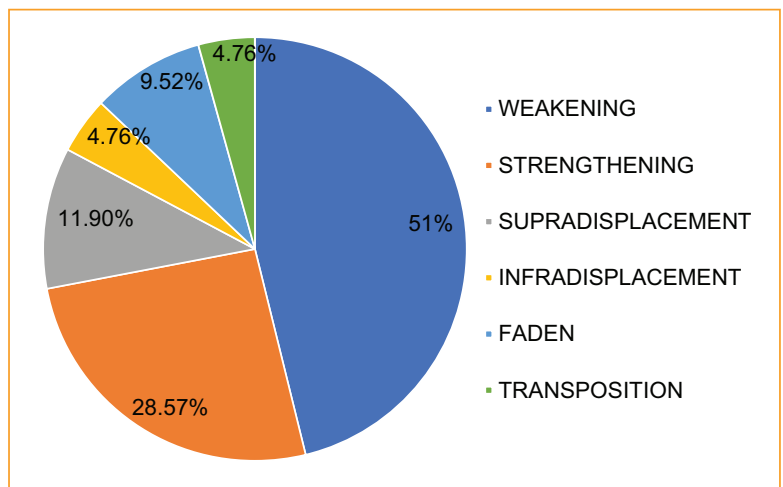

Figure 6. Distribution by the type of procedure performed.

practice is to follow the standard of using a circle or a dot.

Case 3: diagnosis of congenital esotropia with variable angle (Fig. 9)

- Examination:

- Alternate 30-50 DP variable esotropia.

- Ductions and versions: hyperfunction of the inferior oblique muscle of $+1, V$ syndrome, limitation -1 left abduction. DVD +2 left eye.

- Surgical plan: weakening of both internal recti + Faden in left eye internal rectus added to the anterior transposition of the left inferior oblique muscle.

Note: in this patient, the markings on the eye to be operated on look like an " $X$ ", yet today routine clinical practice is to follow the standard of using a circle or a dot.

\section{Scheme:}

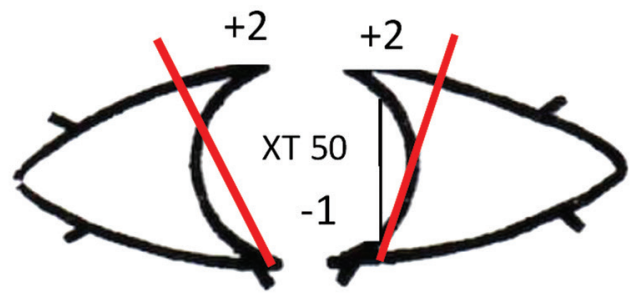

Marking:

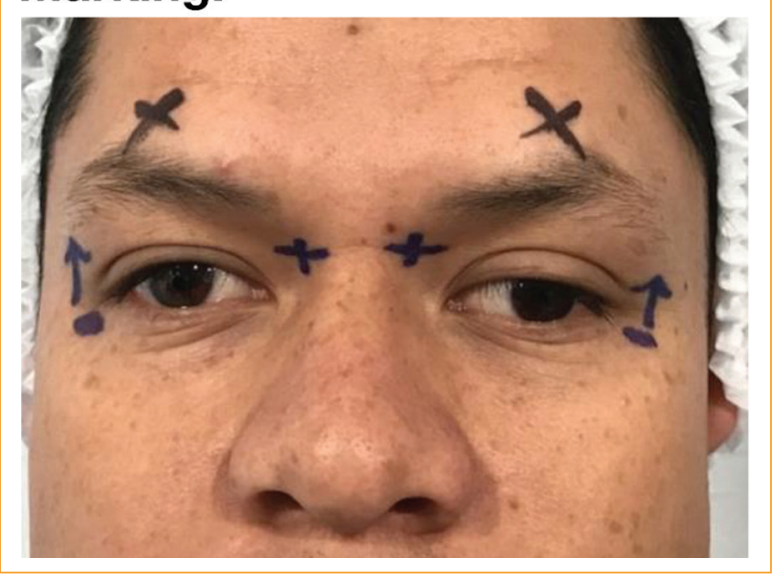

Figure 7. Case 1: diagnosis of right residual monocular exotropia.

\section{Scheme:}

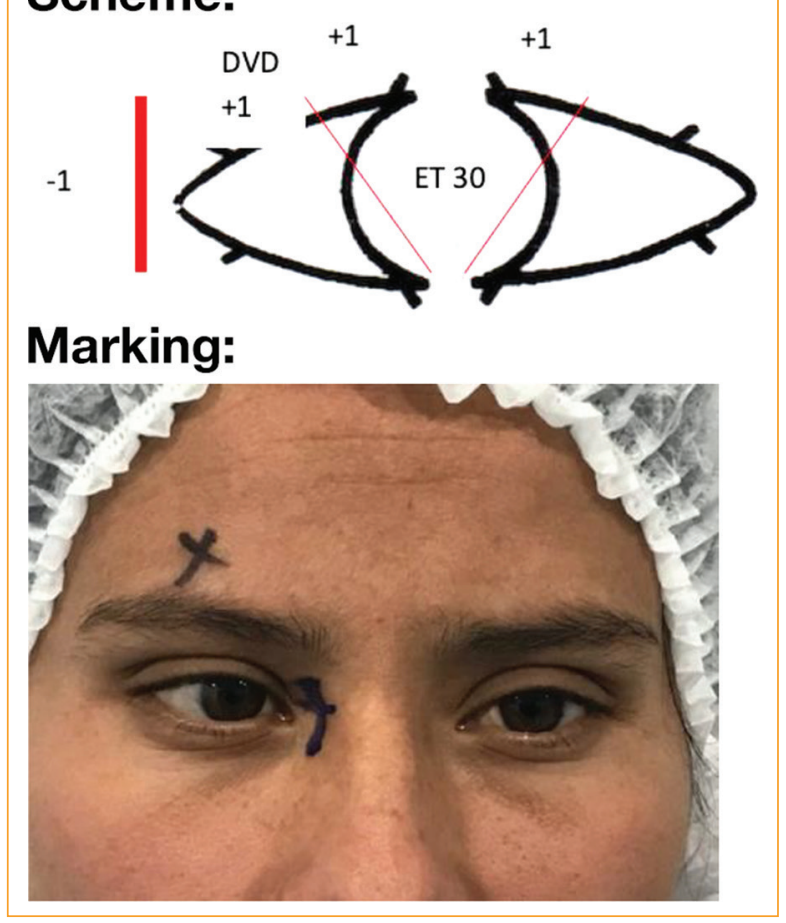

Figure 8. Case 2: diagnosis of right eye monocular esotropia of variable angle. 


\section{Scheme:}

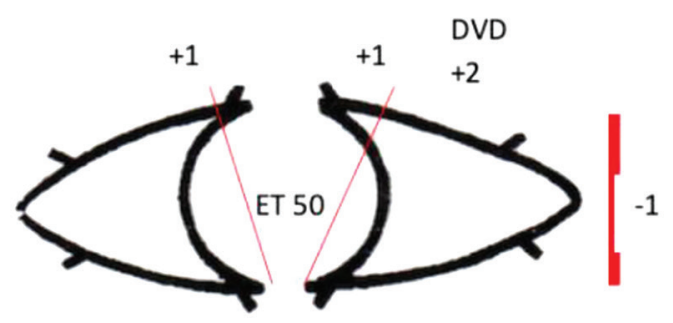

\section{Marking:}

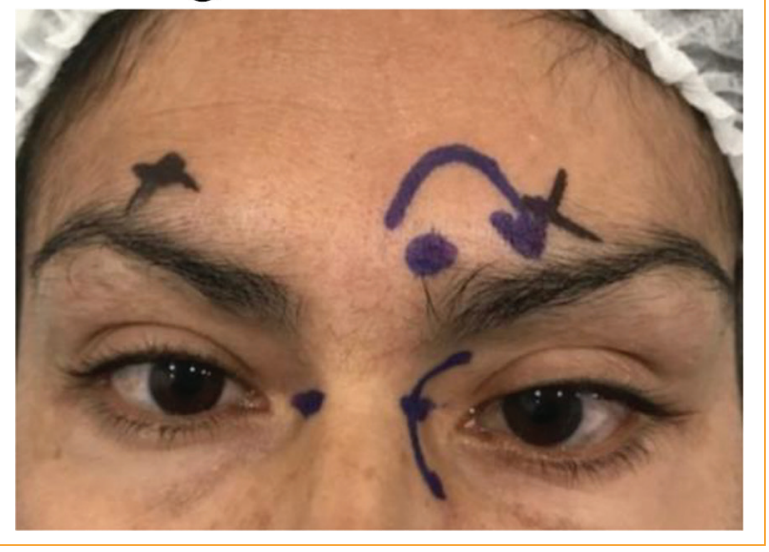

Figure 9. Case 3: diagnosis of congenital esotropia with variable angle.

Case 4: diagnosis of sixth nerve palsy of the right eye (Fig. 10)

- Examination:

- PPM: right monocular esotropia of $>90$ DP.

- Ductions and versions: limitation -4 right eye abduction.

- Surgical plan: weakening of right eye medial rectus + strengthening of the lateral rectus added to hemitransposition of the inferior and superior rectus to the site of lateral rectus.

Case 5: diagnosis of congenital esotropia and $A$ syndrome (Fig. 11)

- Examination:

- 25 DP esotropia.

- Ductions and versions: hyperfunction of superior oblique muscles +3 , A syndrome without incomitance in lateroversion.

- Surgical plan: weakening of both internal recti with supradisplacement.

Note: in this patient, the markings on the eye to be operated on look like an "X", yet today routine clinical practice is to follow the standard of using a circle or a dot.

\section{Scheme:}

$-4$

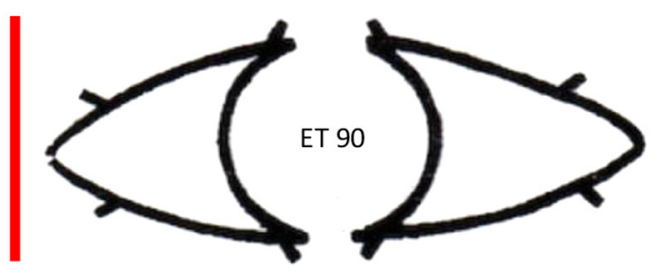

\section{Marking:}

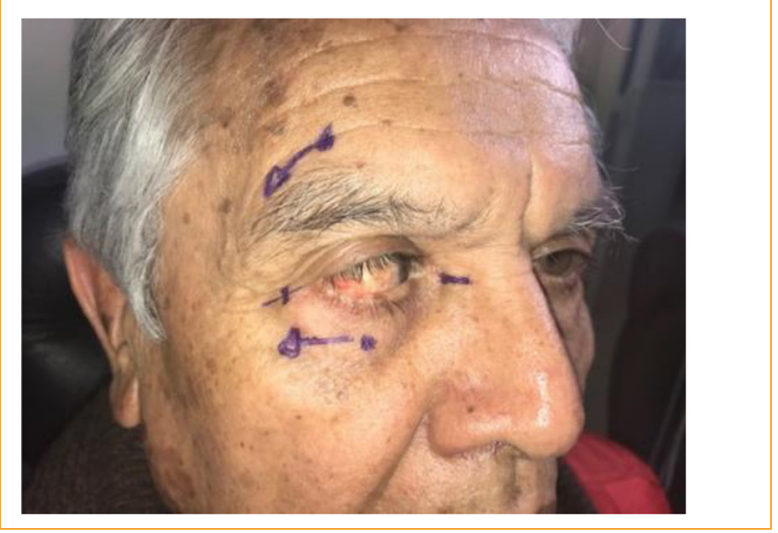

Figure 10. Case 4: diagnosis of sixth nerve palsy of the right eye.

\section{Discussion}

Of a total of 23 patients, women were predominant at a 1.1:1 ratio. In most cases, both eyes were operated on in the same surgical act; muscular weakening was the most common procedure.

The marking procedure was performed without any difficulties in all patients, and it took no more than 5 minutes. We should mention that a fine-tipped permanent black marker should be used so that the mark is not erased during the intraoperative procedure.

It was possible to corroborate the surgical approach in all the cases, and the marking helped during the intraoperative period to carry out the plan that had been pre-established, to not mislead the eye to be operated on in case of asymmetrical surgery, as well as to identify the case fully, above all when several patients are operated on within the same surgical day, thereby reducing the chances of errors ${ }^{5}$.

Marking based on the usual strabological scheme helps it become a universal measure, in addition to the fact that it reinforces knowledge of the muscular actions to be modified with the surgery.

Three months after the protocol was initiated, a survey was conducted consisting of five questions asked to the 


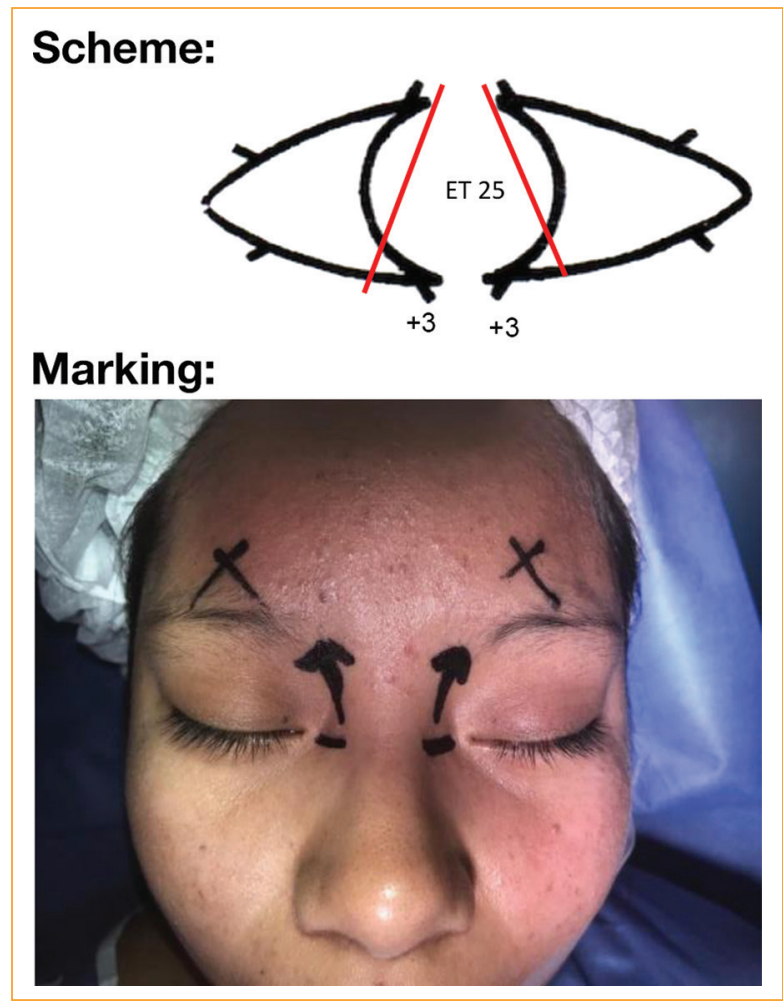

Figure 11. Case 5: diagnosis of congenital esotropia and A syndrome.

surgeon in charge of performing the surgeries and marking of the patients to know about the surgeon's experience and compare it to his previous experience without marking (the questionnaire is annexed to Fig. 12).

When asked about the safety perceived while the surgery was being performed, the surgeon answered that he noticed the difference, being surgery confirmation and surgical plan the most noteworthy of all regarding patients operated on without prior marking.

Regarding the staff involved in the surgery, mainly the residents, the surgeon answered that he noticed a great difference in the attitude and synchronization when the surgical act was performed, as well as reinforcement of teaching regarding pathophysiology, anatomy, and the surgical technique used.

Finally, the expert surgeon recommended the use of marking in all patients treated with strabismus surgery, as the safest measure, which is already part of the preoperative protocol.

\section{Conclusions}

After a bibliographical review, we found that most errors in strabismus surgery are due to operating on the wrong eye or muscle. Following a series of verification
1. Had you ever performed some type of marking prior to surgery on any patients treated with strabismus surgery?
1. Yes
2. No

2. Did you notice any safety differences regarding the performance of the procedure when you marked patients treated with strabismus surgery?

3. Little difference

4. Much difference

5. No difference

3. Did you notice any changes in the nursing or medical staff involved in the surgical event regarding confidence in the procedure to be performed?
1. Little difference
2. Much difference
3. No difference

4. Did you notice any changes regarding communication among the staff involved in the surgical event, resulting into a greater competence by the team?
1. Little difference
2. Much difference
3. No difference

5. Would you recommend marking the surgical site as a guide in strabismus correction surgery in all patients?
4. Yes
5. No
6. In some patients

Figure 12. Questionnaire used to survey the surgeon at the end of the study.

steps prior to the surgical event (eye, number of muscles, and type of surgery for each one of them) minimizes the risk of this type of error. However, when performing the marking, we should make sure that it is done with understandable and legible signs and marks, and most important of all, these signs and marks should be known by all the team members involved in the process and the surgery. We found that when patients are marked prior to the surgery, it is easier for the team to coordinate the procedure, thereby reducing the possibility of errors, in addition to reinforcing teaching, because it allows the surgeon responsible for teaching the resident or physician-in-training what the surgical plan is since marking is performed based on the 
pathophysiology and functional regions of extraocular eye muscles.

We propose this marking technique as part of the immediate preoperative protocol of all patients treated with surgical strabismus correction.

\section{Funding}

The authors declare that for this research no external sources of financing were used to conduct this study.

\section{Conflicts of interest}

The authors did not declare any conflict of interest.

\section{Ethical disclosures}

Protection of human subjects and animals in research. The authors declare that no experiments were performed on humans or animals for this study.

Patients' data protection. The authors declare that they have followed the protocols of their work center on the publication of patient data.
Right to privacy and informed consent. The authors have obtained the written informed consent of the patients or subjects mentioned in the article. The corresponding author is in possession of this document.

\section{References}

1. Maloley L, Morgan LA, High R, Suh DW. Wrong-site surgery in pediatric ophthalmology. J Pediatr Ophthalmol Strabismus. 2018;55:152-8.

2. Ridge RA. Doing right to prevent wrong-site surgery. Nursing. 2008; 38:24-5

3. Shen E, Porco T, Rutar T. Errors in strabismus surgery. JAMA Ophthalmol. 2013;131:75-9

4. DeVine J, Chutkan N, Norvell DC, Dettori JR. Avoiding wrong site surgery: a systematic review. Spine (Phila Pa 1976). 2010;35(9 Suppl):S28-36.

5. Recommendations of American Academy of Ophthalmology Wrong-Site Task Force: 2014. American Academy of Ophthalmology Web site. https:// www.aao.org/patient-safety-statement/recommendations-of-american-academy-ophthalmology. Published August 2014. Accessed August 13, 2015.

6. The Joint Commission National patient safety goals: universal protocol for preventing wrong site, wrong procedure, wrong person surgery. 2011. Disponible en: http://www.jointcommission.org/assets/1/6/2011_NPSGs_ HAP.pdf

7. Mak ST. Sentinel events in ophthalmology: experience from Hong Kong. J Ophthalmol. 2015;2015:454096.

8. Millenson ML. Pushing the profession: how the news media turned patient safety into a priority. Qual Saf Health Care. 2002;11:57-63.

9. Aroichane M. Planning strabismus surgery: how to avoid pitfalls and complications. Am Orthopt J. 2016;66:63-78.

10. Yoon RS, Alaia MJ, Hutzler LH, Bosco JA $3^{\text {rd }}$. Using "near misses" analysis to prevent wrong-site surgery. J Healthc Qual. 2015;37:126-32.

11. Vladuțiu C. [Major steps in strabismus surgery]. Oftalmologia. 2007; $51: 74-8$ 